\title{
Near-Death Experiences Among Survivors of the 1976 Tangshan Earthquake
}

\author{
Feng Zhi-ying \\ Liu Jian-xun \\ Anding Psychiatric Hospital, Tianjin, China
}

ABSTRACT: We interviewed 81 survivors of the severe earthquake in Tangshan in 1976 and found that 32 (40 percent) reported near-death experiences (NDEs) as measured by Greyson's (1983) NDE Scale. The great majority of these NDEs were of the cognitive and transcendental types, and our observations were somewhat different from those of Greyson (1985) in the United States and of Pasricha and Stevenson (1986) in India. These differences suggest that the components, sequences, and types of NDE might differ with race, religion, psychological and cultural background, and kind of near-death event.

Near-death experiences (NDEs), profound subjective events experienced on the threshold of death, have been reported by people who have been seriously injured or ill but unexpectedly recovered, and by people who have anticipated imminent death in potentially fatal situations but escaped from that danger (Feng and Liu, 1986; Greyson and Stevenson, 1980). They represent a type of mental action during the process of dying distinct from other phenomena in the psychology of death.

In December 1987, we conducted a study of 81 survivors of the severe earthquake that had occurred 11 years earlier in Tangshan, China. This paper reports the results of that study.

Feng Zhi-ying is a chief physician, and Liu Jian-xun is a physician-in-charge, in the Department of Psychiatry at Anding Hospital. Reprint requests should be addressed to Dr. Feng Zhi-ying at the Department of Psychiatry, Anding Psychiatric Hospital, De Cai Li, He Xi District, Tianjin 300074, The People's Republic of China. 


\section{Sample}

All subjects in this study were patients in the Paraplegic Convalescent Hospital in Tangshan. They had been severely crushed and in a near-death state when their homes were destroyed in the earthquake, but had been rescued from the danger. We randomly selected 100 patients as investigative subjects. Of those, 19 patients were uncooperative or refused to answer questions.

Of the remaining 81 patients who participated in the study, 43 (53 percent) were men and 38 (47 percent) women. While 5 patients (6 percent) had recovered from their physical injuries, 22 (27 percent) remained partially paraplegic and 54 (67 percent) completely paraplegic.

\section{Method}

In addition to reviewing patients' medical records, we administered to these subjects a questionnaire that contained general demographic and sociological items and questions regarding personality, beliefs and attitudes, and NDE phenomena, including Bruce Greyson's (1983) NDE Scale. Each subject was interviewed by both of us simultaneously, to assure consistency in the ratings, for a period of 3-4 hours. Comparison of our individual ratings on a small preliminary sample of subjects revealed a $95 \%$ consistency rate.

The section of the questionnaire regarding NDE phenomena consisted of a list of $\mathbf{4 0}$ items that we derived from previous reports in the literature and from consultation with experienced investigators. We asked subjects whether or not they had experienced during the earthquake any of the 40 items comprising our phenomenological section. These included (1) feeling estranged from the body, or not recognizing it, as if it belonged to someone else; (2) unusually vivid thoughts; (3) lack of emotions; (4) unusual bodily sensations, such as numbness or heat; (5) life seeming like a dream; (6) a feeling of dying; (7) a feeling of peace or euphoria; (8) a life review or "panoramic memory"; (9) thinking unusually fast; (10) time seeming to go faster than usual; (11) an out-of-body experience; (12) sensation of the world being exterminated or ceasing to exist; (13) a sense of weightlessness; (14) one's self feeling unreal; (15) senses unusually vivid; (16) sudden understanding; (17) seeing deceased or religious figures; (18) thoughts or movements not under conscious control; (19) a feeling of being pulled or squeezed; (20) an unearthly realm of existence; (21) being controlled by an external 
force; (22) senses blurred or dull; (23) ambivalence about death, that is, wanting to proceed into an afterlife but feeling reluctant to leave the earthly world; (24) feeling detached from one's surroundings; (25) being judged or held accountable; (26) the world seeming unreal; (27) time seeming to slow down or stop; (28) visions of the future; (29) a feeling of cosmic unity; (30) a tunnel-like dark region; (31) thinking blurred or dull; (32) a border or point of no return; (33) an unnaturally brilliant light; (34) a feeling of having been dead; (35) extrasensory perception; (36) meaningful sounds; (37) a feeling of joy or pleasantness; (38) meaningful visions; (39) feeling of being a different person or a different kind of person; and (40) unusual scents.

The NDE Scale (Greyson, 1983) includes four distinct components, which in our questionnaire were as follows: the cognitive component included items 8, 9, 10, and 16; the affective component, items 7, 29, 33 , and 37; the paranormal component, items 11, 15, 28, and 35; and the transcendental component, items $17,20,32,36$. On the basis of each subject's report, each item was scored as absent ( 0 points) or as present to a lesser or greater degree ( 1 or 2 points). A subject was judged to have had an NDE if his or her experience scored 7 or more points on the NDE Scale.

\section{Results}

\section{Demographic and Sociological Data}

Subjects' ages at the time of the earthquake averaged 31.4 years (S.D. $=11.3$ ), with a range of 12 to 60 years. Four subjects (5 percent) were younger than 18 years old at that time; $51(63 \%)$ were between 19 and 30 years old; and 26 (32 percent) were older than 30 years. Of the 81 subjects, 79 (98 percent) were Han people and 2 (2 percent) were Moslems.

Forty-two subjects (52 percent) were married, and 39 (48 percent) single. Six subjects ( 7 percent) had no formal education; 19 subjects (23 percent) had an elementary education; 33 (41 percent) had attended junior middle school; 18 (22 percent) had attended senior middle school or obtained secondary technical education; and 5 ( 6 percent) had higher education.

Eleven subjects (14 percent) were administrators or technicians; 27 (33 percent) were laborers; 12 (15 percent) were peasants; and 31 (38 percent) were housewives or educated youth who had followed Chair- 
man Mao's instructions to settle down in the countryside to become the new class of peasants.

\section{Personality}

On the section of the questionnaire describing personality prior to the earthquake, 18 subjects (22 percent) were classified as introverted, 37 (46 percent) as extroverted, and 26 (32 percent) as neither, as determined by Gueng Yue-Xian's Personality Inventory. Forty-seven subjects ( 58 percent) had a change in personality after the earthquake: 2 subjects ( 2 percent) became unrealistically optimistic, 26 (32 percent) became more irritable, and 19 (23 percent) became more docile. These changes were comparable for male and female subjects.

\section{Altered Consciousness}

Some confusion or altered state of consciousness at the time of the earthquake was described by 37 subjects ( 46 percent). That confusion lasted less than an hour for 19 subjects (23 percent of the total), between 1 and 3 hours for 7 subjects ( 9 percent), and more than 3 hours for 11 subjects ( 14 percent). Thirty-seven subjects ( 46 percent) reported having been awakened by the earthquake.

\section{Beliefs and Feelings About Death}

Belief in spirits, ghosts, God, and destiny prior to the earthquake was reported by 22 subjects ( 27 percent).

Regarding their views of death at the time of the earthquake, 25 subjects (31 percent) reported painful feelings about death; 11 (14 percent) reported joyful feelings; 24 (30 percent) felt they would enter an unearthly realm, reunite with deceased relatives, or exist in some other form after death; and 21 (26 percent) had no thoughts about death or believed death would be the end, like a light going out.

Twenty-three subjects (28 percent) had had some knowledge of NDEs prior to the earthquake; and 3 (4 percent) had had a prior close brush with death, though none recalled a prior NDE.

Sixty subjects (74 percent) reported indifference to their experience during the earthquake or spoke freely about it; 18 ( 22 percent) reported fear or pain surrounding their memories; 2 ( 2 percent) reported rage; 
and 1 ( 1 percent) only spoke of it grudgingly. Memories of the experience remained fresh at the time of the interview, 11 years later, or clearer than memories of other events at the same time, for 67 subjects (83 percent).

\section{NDE Phenomenology}

The number and percent of subjects who reported experiencing each of the 40 NDE phenomenological items are listed in Table 1.

\section{NDE Scale}

Of the 81 subjects, 9 reported having experienced none of the 16 items on the NDE Scale, and therefore had scores of 0 . The remaining 72 subjects who reported some phenomena had a mean NDE Scale score of 9.88 (S.D. $=2.79$ ). Thirty-two subjects ( $40 \%$ of the 81 subjects) had NDE Scale scores of 7 or greater, indicating the occurrence of an NDE.

The number of subjects whose responses were rated 2,1 , and 0 for each item on the NDE Scale are listed in Table 2. The highest scoring items were thought acceleration (mean score $=0.64$ ), life review (mean $=0.58$ ), and time acceleration (mean $=0.53$ ) in the cognitive component; peace (mean $=0.56$ ) in the affective component; out-of-body experience (mean $=0.51$ ) in the paranormal component; and visible spirits (mean $=0.56$ ) in the transcendental component.

The mean score of the 81 subjects on the cognitive component was 2.04; on the affective component, 1.01; on the paranormal component, 1.17; and on the transcendental component, 1.30 .

Following Greyson's (1985) categorization of NDEs into types based on the component with the highest score, we classified the 32 NDEs as cognitive in 11 cases (34\%), affective in 1 case (3\%), paranormal in 4 cases (13\%), and transcendental in 9 cases (28\%); the remaining 7 cases (22\%) we could not classify unequivocally.

\section{Correlates of NDEs}

For each of the 40 individual NDE phenomenological items, we looked for significant associations with demographic and personality traits, circumstances of the near-death event, and aftereffects of the 


\section{Table 1}

Frequency of NDE phenomenological items

\begin{tabular}{|c|c|}
\hline Item & $\begin{array}{l}\text { Subjects reporting } \\
\text { item }(n=81)\end{array}$ \\
\hline 1. feeling estranged from the body & $55(68 \%)$ \\
\hline 2. unusually vivid thoughts & $53(65 \%)$ \\
\hline 3. loss of emotions & $51(63 \%)$ \\
\hline 4. unusual bodily sensations & $49(60 \%)$ \\
\hline 5. life seeming like a dream & $47(58 \%)$ \\
\hline 6. a feeling of dying & $46(57 \%)$ \\
\hline 7. a feeling of peace or euphoria & $42(52 \%)$ \\
\hline 8. a life review or "panoramic memory" & $41(51 \%)$ \\
\hline 9. thinking unusually fast & $41(51 \%)$ \\
\hline 10. time seeming to go faster than usual & $35(43 \%)$ \\
\hline 11. an out-of-body experience & $35(43 \%)$ \\
\hline 12. sensation of the world being exterminated & $34(42 \%)$ \\
\hline 13. a sense of weightlessness & $32(40 \%)$ \\
\hline 14. one's self feeling unreal & $27(33 \%)$ \\
\hline 15. senses unusually vivid & $23(28 \%)$ \\
\hline 16. sudden understanding & $23(28 \%)$ \\
\hline 17. seeing deceased or religious figures & $23(28 \%)$ \\
\hline 18. thought, movement not under conscious control & $23(28 \%)$ \\
\hline 19. a feeling of being pulled or squeezed & $23(28 \%)$ \\
\hline 20. an unearthly realm of existence & $21(26 \%)$ \\
\hline 21. being controlled by an external force & $19(23 \%)$ \\
\hline 22. senses blurred or dull & $19(23 \%)$ \\
\hline 23. ambivalence about death & $19(23 \%)$ \\
\hline 24. feeling detached from one's surroundings & $18(22 \%)$ \\
\hline 25. being judged or held accountable & $18(22 \%)$ \\
\hline 26. the world seeming unreal & $17(21 \%)$ \\
\hline 27. time seeming to slow down or stop & $16(20 \%)$ \\
\hline 28. visions of the future & $14(17 \%)$ \\
\hline 29. a feeling of cosmic unity & $13(16 \%)$ \\
\hline 30. a tunnel-like dark region & $13(16 \%)$ \\
\hline 31. thinking blurred or dull & $13(16 \%)$ \\
\hline 32. a border or point of no return & $12(15 \%)$ \\
\hline 33. an unnaturally brilliant light & $12(15 \%)$ \\
\hline 34. a feeling of having been dead & $12(15 \%)$ \\
\hline 35. extrasensory perception (ESP) & $11(14 \%)$ \\
\hline 36. meaningful sounds & $10(12 \%)$ \\
\hline 37. a feeling of joy or pleasantness & $8(10 \%)$ \\
\hline 38. meaningful visions & $7(9 \%)$ \\
\hline 39. feeling of being a different person & $5(6 \%)$ \\
\hline 40. unusual scents & $1(1 \%)$ \\
\hline
\end{tabular}


Table 2

NDE Scale Scores

\begin{tabular}{lrrr}
\hline & \multicolumn{3}{c}{ Subjects $(n=81)$ scoring: } \\
\cline { 2 - 4 } Item & 2 points & 1 point & 0 points \\
\hline Cognitive component: & & & \\
time acceleration & $8(10 \%)$ & $27(33 \%)$ & $46(57 \%)$ \\
thought acceleration & $11(14 \%)$ & $30(37 \%)$ & $40(49 \%)$ \\
life review & $6(7 \%)$ & $35(43 \%)$ & $40(49 \%)$ \\
sudden understanding & $0(0 \%)$ & $23(28 \%)$ & $58(72 \%)$ \\
Affective component: & & & \\
peace & $3(4 \%)$ & $39(48 \%)$ & $39(48 \%)$ \\
joy & $0(0 \%)$ & $8(10 \%)$ & $73(90 \%)$ \\
cosmic unity & $3(4 \%)$ & $10(12 \%)$ & $68(84 \%)$ \\
brilliant light & $1(1 \%)$ & $11(14 \%)$ & $69(85 \%)$ \\
Paranormal component: & & & \\
extrasensory perception & $4(5 \%)$ & $7(9 \%)$ & $70(86 \%)$ \\
unusually vivid senses & $2(2 \%)$ & $21(26 \%)$ & $58(72 \%)$ \\
precognitive visions & $0(0 \%)$ & $14(17 \%)$ & $67(83 \%)$ \\
$\quad$ out-of-body experience & $6(7 \%)$ & $29(36 \%)$ & $46(57 \%)$ \\
Transcendental component: & & & \\
$\quad$ unearthly realm & $2(2 \%)$ & $19(23 \%)$ & $60(74 \%)$ \\
meaningful sounds & $2(2 \%)$ & $9(11 \%)$ & $71(88 \%)$ \\
visible spirits & $22(27 \%)$ & $1(1 \%)$ & $58(72 \%)$ \\
point of no return & $12(15 \%)$ & $0(0 \%)$ & $69(85 \%)$ \\
\hline
\end{tabular}

experience, by assigning numerical values to categorical variables (e.g., "yes" $=1$; "no" =0) and using correlation coefficients. Since this procedure required 40 interdependent tests of significance for each variable, we used the Bonferroni procedure to correct error risk (Grove and Andreasen, 1982). This technique permits the assignment of levels of significance for tests of multiple hypotheses such that the probability of at least one hypothesis being accepted, when in fact the null hypothesis is true for all cases, can be set at an acceptable level. Using the Bonferroni inequality, we set the significance for each individual correlation coefficient at $p=.001$, which corresponds to an overall error rate of $p=.04$ for the family of $40 \mathrm{NDE}$ items.

Using that criterion for statistical significance, none of the $40 \mathrm{NDE}$ phenomenological items was associated with subjects' age; gender; marital status; educational level; occupational level; introversion 
versus extroversion; presence of brain trauma; belief in spirits, ghosts, God, or destiny; or prior knowledge of NDEs.

Alteration in state of consciousness $(\mathrm{r}=.3850)$ and duration of loss of consciousness $(r=.3981)$ were each significantly associated with only one phenomenological item: a feeling of having been dead.

Remaining paraplegic after the earthquake was significantly associated with only one phenomenological item: strange bodily sensations $(\mathrm{r}=.3839)$.

Views of death were assigned the following numerical values: $3=$ belief in an unearthly realm after death; $2=$ joyful feelings about death; $1=$ painful feelings about death; and $0=$ belief that death is the end of existence, or no thoughts about death. Higher scores on this scale of views of death were significantly associated with two phenomenological items: sudden understanding $(\mathrm{r}=.3733)$ and experiencing an unearthly realm of existence $(r=.4402)$.

\section{Discussion}

Since Swiss geologist Albert Heim published a collection of NDEs among falling mountain climbers (1892), many scholars have investigated near-death phenomenology. NDEs seem to follow a consistent pattern, have extensive paranormal contents, and positive transformative effects (Feng, 1986; Feng and Liu, 1986; Greyson and Stevenson, 1980).

\section{Contents of the NDE}

Our subjects reported NDE phenomenological items not mentioned, or rarely mentioned, in NDEs reported from other countries: sensations of the world being exterminated or ceasing to exist, a sense of weightlessness, a feeling of being pulled or squeezed, ambivalence about death, a feeling of being a different person or a different kind of person, and unusual scents. The predominant phenomenological features in our series were feeling estranged from the body as if it belonged to someone else, unusually vivid thoughts, loss of emotions, unusual bodily sensations, life seeming like a dream, a feeling of dying, a feeling of peace or euphoria, a life review or "panoramic memory," and thinking unusually fast.

These are not the same phenomenological features most commonly found by researchers in other countries. Greyson (1983) reported the 
most common phenomenological features of American NDEs to be a feeling of peace, joy, time stopping, experiencing an unearthly realm of existence, a feeling of cosmic unity, and an out-of-body experience. Satwant Pasricha and Ian Stevenson (1986) reported that NDEs in India commonly included seeing a "being of light" or religious figure and being taken to another realm by a messenger and then "sent back." These differences in frequencies of phenomenological items might be associated with race or with religious and cultural background.

\section{Factors Influencing the NDE}

Alterations in state of consciousness, duration of loss of consciousness, and prior views of death influenced the content of NDEs in our series. This suggests that physiological and psychological variables can influence NDE phenomenology. On the other hand, age, gender, marital status, educational and occupational level, personality, brain trauma, and prior knowledge of NDEs and belief in spirits, ghosts, God, and destiny did not significantly affect NDE contents.

\section{Consequences of the NDE}

Greyson and Stevenson (1980) reported that attitude changes were common among NDErs who reported a feeling of being dead and a life review. Our study did not confirm that association. We did find that some subjects, interviewed 11 years after the earthquake, had changes in character.

\section{Types of NDE}

Greyson (1985) reported that the nature of the near-death event may influence the type of NDE. He reported that NDEs were generally of the affective or transcendental type in circumstances in which death might have been anticipated, such as suicide attempts, exacerbations of chronic illness, or complications of surgery; but that in situations in which the NDE was sudden and unexpected, roughly equal numbers of experiences were cognitive, affective, and transcendental.

The near-death event in our study was a sudden and unanticipated earthquake. Of those NDEs that we could categorize as to type, the 
most common type was cognitive, followed by transcendental, and only one NDE was of the affective type. This distribution of types differs from that reported by Greyson (1985) for unexpected events in that we found virtually no affective experiences. The preponderance of cognitive NDEs in our series supports the argument (Stevenson and Greyson, 1979; Greyson, 1985) that such experiences are more common in sudden and unexpected events.

\section{Conclusion}

Near-death research has practical significance for social medicine and clinical psychiatry (Feng, 1987); NDEs appear to exert positive transformative effects once subjects are out of danger. The phenomenological content of the NDE furthermore has implications for psychology, sociology, and eschatology, as well as medical and other sciences. Our study was preliminary and exploratory, and dealt with the results of one specific calamitous near-death event; hence we must be cautious about generalizing from these data to other populations. We intend to pursue these investigations with other high-risk populations to eliminate the biases inherent in the present sample.

\section{References}

Feng Zhi-ying. (1986). [NDE has positive transformative effects on the individual in danger.] Medicine and Philosophy, 3, 36-37.

Feng Zhi-ying. (1987). [Research into near-death experiences in foreign countries.] Medical Information in Tianjin, 2, 19-21.

Feng Zhi-ying and Liu Jian-xun. (1986). [A research into present condition of near-death experiences.] Referential Journal of Psychiatry, 13, 85-89.

Greyson, B. (1983). The Near-Death Experience Scale: Construction, reliability, and validity. Journal of Nervous and Mental Disease, 171, 369-375.

Greyson, B. (1985). A typology of near-death experiences. American Journal of Psychiatry, 142, 967-969.

Greyson, B., and Stevenson, I. (1980). The phenomenology of near-death experiences. American Journal of Psychiatry, 137, 1193-1196.

Grove, W. M., and Andreasen, N. C. (1982). Simultaneous tests of many hypotheses in exploratory research. Journal of Nervous and Mental Disease, 170, 3-8.

Heim, A. (1892). Notizen uber den Tod durch Absturz [Remarks on fatal falls]. Jahrbuch des schweizerischen Alpclub, 27, 327-337.

Pasricha, S., and Stevenson, I. (1986). Near-death experiences in India: A preliminary report. Journal of Nervous and Mental Disease, 174, 165-170.

Stevenson, I., and Greyson, B. (1979). Near-death experiences: Relevance to the question of survival after death. JAMA, 242, 265-267. 